\title{
THE GHANA HIGHWAY CODE AS A TEACHING AND LEARNING MATERIAL
}

\author{
N. K. Nsiah-Achampong*, I. K. Yankson, W. K. Agyemang And N. A. Mingle \\ (N. K. N-A, I. K. Y. \& W. K. A.: CSIR - Building and Road Research \\ Institute (BRRI), Kumasi, Ghana; N. A. M.: CSIR - Institute of Scientific \\ and Technological Information (INSTI)). \\ *Corresponding author's email: n.nsiah@inorbit.com
}

\begin{abstract}
The Ghana Highway Code is the official state-recognised traffic teaching and learning material widely used in traffic schools and by motorists in Ghana. Ghana changed its traffic from left-hand to right-hand drive in 1974; the Code was subsequently published to, besides other reasons, guide road users to be reconditioned to a fresh standard of behaviour. However, 46 years after its publication, the content of the Code has been outgrown by new developments in the road environment and traffic systems, rendering the Code ineffective. Content analysis was used to ascertain the validity and reliability of the Code. Out of 95 motorists interviewed, $91.5 \%$ indicated they use or had ever used the Code, while $3.2 \%$ had not used it before and 5.3\% did not know about it at all. Hundred percent of driving schools used the Code as teaching and learning material. It was concluded that the Code was an obsolete teaching and learning material. The implication was that, the wide user population of the Code had an adverse impact on learners. It was recommended that the Code be revised and republished as new edition.
\end{abstract}

Keywords: Ghana Highway Code, Educand, Road Environment, Teaching, Learning.

\section{Introduction}

The Ghana Highway Code is the official state-recognised and most widely patronised book for motorists in Ghana. It was published and promulgated in 1974 when Ghana changed its traffic system from left-hand to right-hand drive. The objectives of the Code include teaching road users how to drive, ride or walk safely on the roads to avoid road accidents; serve as a standard of behaviour to all road users; and serve as a source of guidance to all road users (Ministry of Roads and Transport (MORT), 1974). The rationale behind the Code is the attainment of road safety by the strict compliance of its provisions by motorists and pedestrians. The Code is patronised widely by all of road users in Ghana. The identified users and educands of the Code include the Ghana Education Service (GES), driving schools, learner drivers, practising drivers, the police and pedestrians.

Published in 1974, the Code is 46 years old (1974 - 2016). Currently, the transport system and road environment in Ghana have undergone major transformations and developments, making the Code relatively obsolete. The Code had therefore become operationally ineffective. There are many reprints of the Code in circulation with variations in content. These are, however, not marked as 
different editions to meet publishing requirements. This makes it difficult for the educand to form a basis for determining the most recent and therefore current publication. Researchers are faced with the additional problem of referencing. A piece of text used for analysis may not be traced by someone else using the same references. This is because the same references of folio correspond commonly to all other reprints of the Code irrespective of changes in page content. The study aimed at determining the validity and reliability as a teaching and learning material for road safety education.

\section{The setting}

\section{Experimental}

The study was carried out in Kumasi where the study population was drawn from. Kumasi is the capital town of Ashanti Region. It is located on latitude $6041^{\prime} \mathrm{N}$, longitude 1036'W and has a population of 1,170,270 (Ghana Statistical Service 2002). It is the most populous town in the Region and the second largest city in Ghana after Accra, the national capital. Kumasi is a nodal settlement with major roads passing through it to other regions. Kumasi is linked directly to Accra and Cape Coast in the South, and Sunyani and Tamale in the North.

\section{Data collection and study variables}

The research design was delimited to qualitative research, where the quality of the phenomenon under study, validity of the Ghana Highway Code, was examined. Content analysis was used as a major instrument to bring out content-details of the Code. The units of analysis are authenticity (where authenticity is defined in terms of recognition by International Serial Book Number); and medium variables. Using Medium Variables as expounded by Wimmer and Dominick (1987), the study formed / used seven units of enquiry as Medi- um Variables for Content analysis. These are: (a) unfamiliar technical terms, (b) inadequate information on everyday traffic use, (c) text - ground data inconformity, (d) text - illustration references, (e) Shadowy illustrations, (f) doubtful emergency instructions, (g) discourse problems. Motorists and educands were interviewed to ascertain level of patronage, user-satisfaction and comprehension of the Code.

\section{Sampling}

Interviews were conducted among 12 driving schools; and 95 motorists from different categories, namely commercial vehicle drivers, private vehicle drivers, armed forces vehicle drivers and government vehicle drivers, to ascertain the user domain magnitude of the Code. The population of driving schools in Kumasi was 24 as obtained from the Faith Progressive Driving school, Ashanti Regional representative of the Ghana National Association of Driving Schools. Fifty percent of the schools were selected by systematic sampling in order of the list. Using the first school as first count, every second school was selected. Driver- respondents were selected by purposive sampling.

\section{Results and discussion}

Motorists' awareness of the Ghana Highway Code

Out of 95 motorists interviewed, 87 respondents, representing $91.5 \%$ indicated that they use or had ever used the Code. Three respondents $(3.2 \%)$ had not used the Code before, and five respondents (5.3\%) did not know about the Code at all (Fig. 1). Out of 12 driving schools studied, $100 \%$ indicated that they use the Code as a teaching material. This shows that the Code is highly patronised in Ghana by motorists and training instructors. The situation shows how ready the Ghanaian 
motorist is, to learn. Invariably the driver had responded to the constant urge by traffic authorities to study the Code (Donkor, 2002).

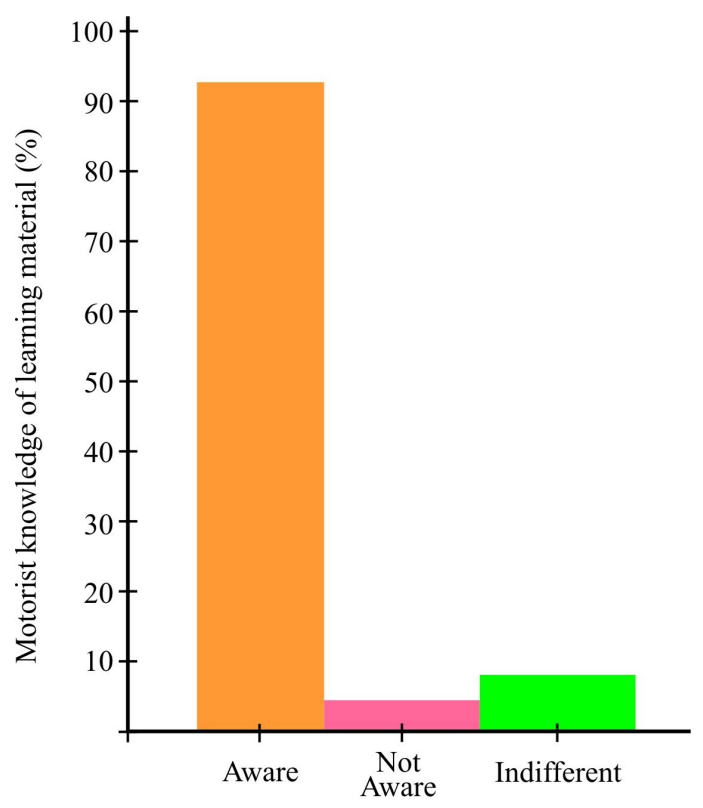

Respondents

Fig. 1: Motorists' awareness of the Ghana Highway Code

\section{Presentation of Medium Variables}

\section{Unfamiliar Technical Terms}

The Ghana Highway Code contains technical terms, which are not commonly used in everyday language. Some of the terminologies are commonly exclusive terms in the traffic domain but are used without working definitions to indicate their contextual meanings. In publications that contain technical words or words with multiple meanings, such words are explained in a glossary to establish an objective line of thought from reading the text. The technical terms used in the Code are as follows: kerb, central islands, traffic subways (MORT, 1974), kerb drill. (MORT, 1974), central reserve (MORT, 1974), expressways, hard shoulder (MORT, 1974), central reverse (sic) (MORT, 1974), slip roads; (MORT, 1974), street refuge or central reservation (MORT, 1974), verges (MORT, 1974).

\section{Inadequate Information on Everyday Traffic Use}

A driver goes through three frequent processes in the course of driving. These are stopping, waiting and parking. The three are problem areas of traffic to both the driver and Traffic Law Enforcement Agents (TLEAs) especially in urban areas where the density of traffic is high. These driving restrictions are not explained in the Code to show the differences among the three. An attempt is made at explaining what "No Waiting" and "No Parking" mean (MORT, 1974): "No Parking" is unequivocally explained to mean same as the "No Parking Sign".

It is not clear what is meant by "not letting your vehicle stand" (MORT, 1974). The educand's understanding of no parking, or not letting vehicle stand is interfered with, with the introduction of the "Clear Way" sign in the discourse. This is because the Code itself does not show what a clear way sign is among the list of signs displayed on pages 32 to 39 . The educand can only make a guess if the "Road Clear" (MORT, 1974) is the same as "Clear Way". In another development, "Stopping" and "Parking" are given (MORT, 1974) only as precautions being part of the general driving principles on the motorway (MORT 1974). 


\section{Text - Ground Data Inconformities}

Some text information in the Code does not match corresponding realistic data on the ground. This creates incongruity in the objective understanding-by-application method of learning. That is, when the educand wants to apply information in the Code to what is in reality, he finds the visual complement to be untrue. Examples of such unconformities are:

"Yellow lines on the road guide, warn, or give orders" (MORT, 1974).

"Do not cross double unbroken yellow lines in the middle of the road (MORT, 1974).

"Keep between the traffic lane markings the short broken yellow lines which divide the road into lanes" (MORT, 1974).

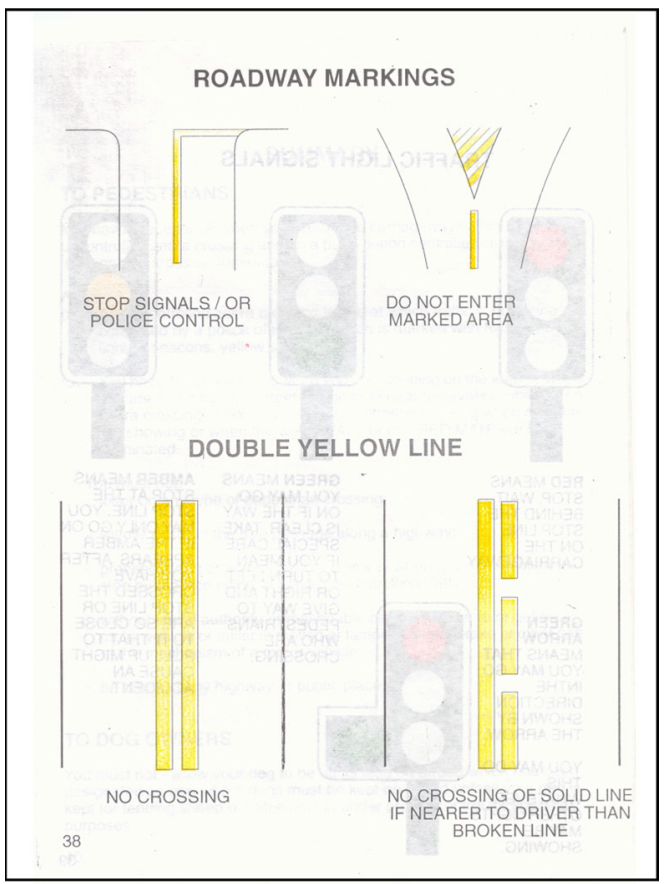

Fig. 2: One of the illustrations on pages 36 to 38 of the Code showing yellow lines instead of white.

\section{Text - Illustration References}

One text reference (MORT, 1974) does not match corresponding folio on pages 39 to 42 as indicated. The reference may either be referring to pages 29 to 39 or 32 to 39 .

\section{Shadowy Illustrations}

Illustrations in the Code are made up of 153 drawings on 20 pages, and one picture on one page. The drawings though illustrate the text messages, there is however, more room for quality improvement. For example, in Figure 3 , the picture (MORT, 1974) does not illustrate the text information definitely because it is unclear. The picture illustration is a combination of Half Tone Copy and Line Copy. The combination is not skillfully done to simulate both in one whole piece. The warning triangle appears independent of the black mass above it. It may take the educand some guesswork to identify all the elements in the black mass.

\section{Doubtful Emergency Instructions}

The Ghana Highway Code gives instructions on control measures in times of emergency. One of them is as follows:

"If your acceleration pedal jams. (sic) turn off the ignition and brake to a stop, leaving the vehicle in gear". (MORT, 1974).

Inversion is found in the emergency instruction stated above. The disadvantage of inversion in language is that it changes the sequential order of items in a message. According to Ewles and Simnett (1996), it is even more serious in a verbal message when a word link such as "after" is not heard by the listener. Similarly, in the emergency instruction given above, the inversion used makes the sequence appear as follows: 
(1) turn off ignition

(2) Step on brakes

(3) Leave vehicle in gear.

The same instruction could be put this way if the inversion is avoided:

"If your acceleration pedal jams, turn off your ignition, leave the vehicle in gear and brake to a stop".

In this option, the sequence becomes:

(1) turn off ignition

(2) leave vehicle in gear

(3) step on brakes.

From 1974, when the Code was published to date, vehicles have undergone mechanical transformations. In the 1970 s, vehicle control systems such as the braking and steering mechanisms were predominantly manually operated and would work when the ignition is turned off.

On the contrary, energy-powered controls have enormously replaced the obsolete manual system in the contemporary era. Brakes are now servo-assisted systems. Hydraulic steering is gradually giving way to powered-steering. For these types of vehicles, when the ignition is turned off, the steering and braking systems cease functioning. Another type of vehicle, the automatic gear system, is not catered for in the emergency instructions.

If the brake does not function when the ignition is turned off, the alternate mechanism of bringing the vehicle to a stop is the gear in motion.

\section{Language ambiguities}

Language is a subjective and sensitive subject that requires precision in composition to carry the intended meaning. In the Code, language seem to be so hurriedly composed that they are beset with omissions, typographical errors and ambiguity that disturb the meaning of information.

Nine omissions on eight pages and 18 typographical errors on 13 pages were identified in the Code. The educand has to imagine a substitute for the omission, and make a probable guess for the typographical error. The academic (or school education) background of the target group for the Code is varied, from holders of the Middle School Leaving Certificate to PhD (Nsiah-Achampong, 2004). Thinking abilities are also in varied degrees. The sociological perceptions of people differ from one person to another. A strict-minded perfectionist may not notice an omission, or typographical error, or ambiguity as such and may take its meaning just as it appears. If each educand has to make an individual guess or personal substitution, different meanings will be assigned to information in the Code and road user attitudes will not be tailored along definite, common lines. This is because the absence of, or the wrong insertion of even a punctuation mark or an article, can affect the meaning of a sentence. An example is as follows:

"You get into a rear wheel skid; do NOT brake Suddenly, turn your steering wheel in the direction of the skid. As the vehicle begins to straighten out, straighten thie (sic) front wheel also". (MORT, 1974).

In this statement, it is not clear if the uppercase "S" in "Suddenly" suggests that there is a full stop at the end of "brake" which has been 
omitted. If there is not, then the word "Suddenly" becomes a modifier that affects the action of braking. If there is no full stop at the end of "brake", then the modifier "Suddenly" affects the turning action of the steering wheel. Braking or braking suddenly, in precision required performances, are two entirely different actions; so are, suddenly turning steering wheel or turning steering wheel. This way, the Ghana Highway Code becomes a teaching and learning material that leaves the learner in a doubtful state of traffic knowledge.

Another problem about the grammatical errors in the Code is the effect it has on pupils in the primary schools. Excerpts are taken from the Code and used as English reading material for pupils in the primary school (Curriculum Research and Development Division, 2001). The rationale behind this is that the pupils will be learning English Language and be acquiring traffic knowledge at the same time. If such errors form the basis of learning for the young brain

in the primary school, then a poor foundation is being created for the learning of English Language. Ferris (1999) points out that linguistic errors made by students learning English as second language are bothersome and affect their overall evaluation. Wornyo (2016) reiterates the essence of English grammar in one's progress in academic work to the extent that poor language proficiency affects the performance of students in other disciplines and affect academic progress.

\section{Omissions}

The following are omissions identified in the Ghana Highway Code. (An omission is indicated by an underscore):

“... where there are slower vehicle_in the lane when you have passed them". (MORT, 1974).
"... or the driver behind you wants_overtake, you should stop at a passing place." MORT, 1974).

"Box junction_have Criss-cross yellow lines painted on the road". (MORT, 1974). “... Be particularly careful about cyclist_” (MORT, 1974).

“...The sign should be place_at least 50 yards (46 metres) before the obstruction, and on the same side of the road ... (MORT, 1974).

“... keep any animal in your change in the vehicle or under proper control_the verge" (MORT, 1974).

"... use a motorway if you are_learner driver" (MORT, 1974).

“... Remove casualty from immediate danger if_likelihood of fire form spilled petrol. (MORT, 1974).

"Stopping distance increase_greatly with wet and slippery roads, whole number" (MORT, 1974)

\section{Typographical errors}

The following are typographical errors identified in the Code. (An error is underlined): "You must give way once they have stopped unto the crossing ..." (MORT, 1974). "Unless road makings indicate otherwise ..." (MORT, 1974).

“... or when joining if from a side road ..." (MORT, 1974).

"... if you see warnings signs and the flashing lights ..." (MORT, 1974). 
“... a speed of 50 m.p.h. or 81 km.p.h may fell like" (MORT, 1974).

"Never stop on or immediately beyond and any level crossing" (MORT, 1974).

"You must not - allow your dog to be off its lead ... unless your dog is kept for tending sheep or cattle or is in under proper control for sporting purposes". (MORT, 1974).

“... not stop your vehicle within the limits of a pedestrians crossing ...” (MORT, 1974).

“... your use of the vehicle is properly insured and that there are no restrictions in the relevant insurance policy (eg. as to who drive)" (MORT, 1974).

"... sound your horn While stationary" (MORT, 1974).

"... keep any animal in your change in the vehicle or under proper control the verge" (MORT, 1974).

“... Remove casualty from immediate danger if likelihood of fire form spilled petrol". (MORT, 1974).

\section{Conclusions}

In its current state, the Ghana Highway Code has outlived its purpose; published 46 years ago, new developments in the road environment and traffic systems have rendered the Code obsolete.

Some errors in the Code set a precedent for arbitrary substitutions by the learner.

It is not clear what the marking scheme for DVLA examination indicates as correct answers to questions relating to lines on the road, and in other areas where learners make arbitrary substitutes.

\section{Recommendations}

Found to be obsolete, the Ghana Highway Code should be republished professionally by the DVLA. The new version should take care of contemporary traffic situations and eliminate language problems associated with the current issue.

Graphics in publishing is now enhanced by technology as compared with graphics in 1974 when the Code was published. The quality of graphics in the new edition should be improved to reflect contemporary times.

\section{References}

CURRICULUM RESEARCH AND DEVELOPMENT DIVISION (2001) Teaching Syllabus for English (Primary school). Ministry of Education, Accra, Ghana. pp.113 - 114.

Donkor, S (2002) Road Users Advised to Study Highway Code. Daily Graphic, January 12, 2002. p.18.

Ewles, L. \& Simnett, I. (1996) Promoting Health. A Practical Guide. Bailliere Tindall, London. p.148.

Ferris, D. R. (1999) The case for grammar correction in L2 writing classes: A response to Truscott (1996). Journal of Second Language Writing 8, 1 - 10.

GHANA STATISTICAL SERVICE (2002) 2000 Population and Housing Census. Special Report on 20 Largest Localities. p.42.

MINISTRY OF ROADS AND TRANSPORT (1974) Ghana Highway Code. Right Hand Traffic pp. $1-47$ 
Nsiah-Achampong, N. K. (2004) The Impact of Traffic Education in Ghana. The Case of Selected Geographical Areas in Ashanti Region of Ghana. Unpublished Thesis submitted to the School of Graduate Studies, KNUST, Kumasi. p. 278

Wimmer, R. D. \& Dominick, J. R. (1987) Mass Media Research. An Introduction:Wadsworth Publishing Company. Belmont, California. pp.167, 497
Wornyo A. A. (2016) Attending to the Grammatical Errors of Students using ConstructiveTeaching and Learning Activities. Journal of Education and Practice 7, (7).

Received 21 Jan 20; revised 07 Aug 20. 